\title{
Methodological details on a published study on red yeast rice and berberine
}

\author{
C. Benvenuti* \\ Medical Consultant Meda Group, Monza, Italy
}

In view of including the data of the study "Clinical evidence of efficacy of red yeast rice and berberine in a large controlled study versus diet" by Trimarco B, Benvenuti C, et al. Mediterr J Nutr Metab (2011) 4:13-19, in an international based meta-analysis, it was judged appropriate, for reason of total transparency, to add more information concerning the methods that were applied. These amendments to the paper, are based on the operative procedure contained in the official protocol "Controlled clinical study on Armolipid Plus, food supplement active on cholesterol and triglycerides in CV risk and metabolic syndrome control".

The study concerns a food supplement, a class of products that usually is not (or scarcely) documented as not required for marketing authorisation. In Methods section the basic criteria of the study design were reported, representing the condition of the Italian real world, to obtain reliable clinical data on efficacy and safety.

The protocol was delivered to the centres accompanied by a case report form in which both activity and safety parameters could be reported. A specific section was dedicated to adverse events description.

Design of the study: due to the huge amount of the involved centres (248), a double blind placebo type was not judged practically feasible from the operative point of view: providing double blind supplies for up to 16 weeks in so many centres requires an extraordinary logistic effort, whose benefits could be considered marginal versus the planned large amount of data to collect.

In fact, the main end point was represented by objective measures (blood lipids) that were determined by laboratories working under the normal routine and not aware of the treatment followed by the subject, allowing to consider the design as "functional double blind".

Each centre was provided a balanced randomisation list (random ratio 2:1 was accepted), and the centres that did not complied with it, were discarded to avoid bias in the comparison.

The protocol fixed as 8 weeks the minimum duration of treatment. Concerning the duration of the treatment, it should be specified that: 8 weeks was the minimal duration of treatment on which the sample size was calculated, and the centres where left free to extend the treatment up to 16 weeks according to the attained result and the availability of the subject to continue it.

The planned 8 weeks' visit was respected by most of the subjects: 707 out 933 (75.8\%) in diet group and 786 out $818(96.1 \%)$ on AP for total cholesterol.

Part of the centres evaluated the parameters even at 4,12 and 16 weeks.

The type of real world study and the inclusion of centres homogeneously spread along Italy, explains the differences in the final number of exams performed at same visit, as each doctor applied his habit to keep under control this type of subject.

\footnotetext{
${ }^{*}$ Corresponding author: Dr. Claudio Benvenuti, via Pier della Francesca 54, 20154 Milan, Italy. Tel.: +39 3351024156; E-mail: benvenuti.cla@gmail.com.
} 
The management of the study encouraged more the participation of the doctors and the subjects, in order to collect as many clinical data and privileged the primary end point (total cholesterol at 8 weeks and adverse events reporting).

The analysis of the data was performed using all the data available, as making calculation on a larger basis avoided losing information or discarding useful data and increased the knowledge on the product. Consequently, the data available at each time for each parameter, were compared with the paired baseline values, and the statistical analysis was conducted time by time between the calculate differences of the groups of treatment.

In conclusion, the study produced robust data, because it was carried out in conditions better resembling the usual daily clinical practice, without a rigorous selection of participating centres, and with simple enrolment criteria, i.e. abnormal cholesterol values and no need or unfeasibility to give a prescription drug.

The laboratory analyses for lipid determination were performed by the individual laboratories of usual reference for the doctors involved that routinely base their prescriptions on these lab values. For this reason and for the planned lack of diet standardisation, the outcomes of the study can be more widely extrapolated. 